\title{
Simulation and Analysis for Electric Bicycle Traffic Flow
}

\author{
Cuiqin Wü, Yinfeng Wu and Xianyan Kuang \\ School of Mechanical and Electrical Engineering, Jiangxi University of Science and Technology, \\ Ganzhou, Jiangxi, China \\ cuiqinwu@163.com \\ * Corresponding author
}

Keywords: traffic engineering, electric bicycle flow, lane changing rule, cellular automaton model. Abstract. The electric bicycle has become the main part of non motor vehicles in small and medium-sized cities. Research on the traffic flow characteristic of the electric bicycle has important practical significance. Based on NaSch model, this paper models electric bicycle traffic flow with $\mathrm{CA}$ model and improves the lane changing model. Then the electric bicycle lanes change into general lane change and whistle change, and corresponding lane changing rules are set up. Simulation analysis of the model is carried out. The results show that when the traffic density is small, whistling behavior to raise the road utilization rate has some effect, but in the high density, whistle behavior can not improve road traffic capacity.

\section{Introduction}

In urban traffic, especially in the medium and small cities, non motorized vehicles are important means of transportation for urban residents. In recent years, the non motor vehicles have changed greatly, which is mainly characterized by the increase of the proportion of electric bicycles in non motorized vehicle traffic, and some even accounts for the vast majority of non motorized vehicles in small and medium cities. The study of traffic flow characteristics of non motorized vehicle, which is composed of electric bicycles, has important practical significance.

Because of the great difference between the non motorized vehicle and the motor vehicle, the traditional methods for motor vehicle modeling such as following model based on dynamics are often difficult to adapt to the modeling of non motor vehicle traffic flow. In recent years, the cellular automaton (CA) model has been widely used in traffic modeling and simulation research.

The first traffic flow CA model is proposed by Nagel and Schreckenberg[1], called NaSch model, which can simulate the actual traffic situation to a certain extent. Base on NaSch model, many improved models[2-5] are built by the domestic and foreign scholars, so that the models can better describe the nonlinear phenomena in traffic.

In recent years, the research on mixed traffic flow(the flow mixed with bicycles) is active. A mixed bicycle traffic model [6] is built using the cellular automata (CA) method to model the characteristics of bicycle passing events in mixed bicycle traffic on separated bicycle paths. A cellular automata model[7] for mixed motorised and bicycle traffic, with agent-based update rule specification, is applied to a 16-node network of one-way roads. The dynamic behaviors of mopeds and bicycles are described using cellular automata method in[8], and the traffic flow model under the interaction between mopeds and bicycles is established.

In this paper, the CA model is used to simulate the electric bicycle flow. Considering the variety of electric bicycle lane changing, the electric bicycle lane changing are divided into general lane changing and whistle lane changing, and corresponding lane changing rules are set up. Simulation analysis of the model is carried out.

\section{Model}

Basic parameters. Cellular automaton consists of 5 basic elements, such as cellular, cellular state, cellular space, neighbor and evolution rule. 
Cellular. According to the physical characteristics of electric bicycle, electric bicycle cellular is defined as a rectangular of the length of 2 meters long, 1 meters wide.

Cellular State. Each car cellular is empty or occupied by a vehicle, electric bicycle speed is $\left(0-v_{\max }\right)$, of which $v_{\max }$ is the maximum speed. According to the actual traffic survey, $v_{\max }=3 \mathrm{cell} / \mathrm{s}$.

Cellular Space. Define the cellular space of the non motor vehicle lane as a net range composed of $1000 * 3$ cells.

Neighbor. According to the actual traffic, the running state of the electric bicycle generally influenced by the vehicles around, therefore the cellular neighbors in model are the non empty cellulars around the cellular .

Evolution Rul. The driving process of electric bicycle is generally regarded as two movements of changing lane and driving forward. Therefore, this model will divide the evolution rules of electric bicycle cellular into lane changing rule and forward rule.

Lane Changing Rule. According to the electric bicycle riding experience, when people blocked by vehicle before and it is safe enough with rear vehicle distance to change to target lane, people will generally choose to change lane. In addition, through observation, non motorized vehicles often spontaneously formed a fast slow lane, fast cars run on the left side of the road. When a fast vehicle is blocked by a slow vehicle on lane, the rider may urge the slow vehicle to change lane through the whistle.

Considering the above characteristics, the lane changing behavior is divided into general lane changing behavior and whistle changing behavior. Define the corresponding change rules according to lane changing characteristics:

General Lane Changing Rule. If the state of electric bicycle is as Eq. 1, then it change to right side lane with probability $p_{\text {change }}$; if the state is as Eq. 2, then change to left side lane with probability $p_{\text {change }}$.

$$
\begin{aligned}
& d_{n}^{f}<\min \left(v_{n}+1, v_{\max }\right) \text { and } d_{n}^{r f}>d_{n}^{f} \text { and } d_{n}^{r b}>v_{n}^{r b} \\
& d_{n}^{f}<\min \left(v_{n}+1, v_{\max }\right) \text { and } d_{n}^{l f}>d_{n}^{f} \text { and } d_{n}^{l b}>v_{n}^{l b}
\end{aligned}
$$

Where, $d_{n}^{f}$ is the distance between the vehicle $n$ and the front vehicle in the same lane; $d_{n}^{r f}\left(d_{n}^{r b}\right)$ is the distance between the vehicle $n$ and the front(back) vehicle in the right side lane; $d_{n}^{l f}\left(d_{n}^{l b}\right)$ is the distance between the vehicle $n$ and the front(back) vehicle in the left side lane; $v_{n}$ and $v_{\max }$ are the speed and the maximon speed of vehicle $n ; v_{n}^{r b}$ and $v_{n}^{l b}$ are the speed of right back vehicle and left back vehicle.

Whistle Changing Rule. If the vehicle does not meet the general lane changing rule, but meet all the conditions of Eq. 3 and Eq. 4, then the vehicle change lane with probability $p_{\text {change }}$.

$$
\begin{aligned}
& h_{n-1}=1 \text { and } d_{n}^{f}>\min \left(v_{n}+1, v_{\max }\right) \text { and } d_{n}^{l f}>\min \left(v_{n}+1, v_{\max }\right) \text { and } d_{n}^{l b}>v_{n}^{r b} \\
& h_{n-1}=\left\{\begin{array}{lll}
1, & \text { if } & d_{n-1}^{f}<\min \left(v_{n-1}+1, v_{\max }\right) \text { and }\left(d_{n-1}^{l f}<d_{n}^{f}\right) \text { and } \\
0, & \text { others }()<p_{w}
\end{array}\right.
\end{aligned}
$$

Where, $h_{n-1}$ is the whistle state of vehicle $n-1, h_{n-1}=1$ shows whistle, $h_{n-1}=0$ shows no whistle.

\section{Update Rules.}

Acceleration: $v_{n}=\min \left(v_{n}+a, v_{\max }\right)$.

Deceleration : $v_{n}=\min \left(v_{n}, d_{n}^{f}\right)$.

Randomization: $v_{n}=\max \left(v_{n}-1,0\right)$, with the randomization probability $p$.

Update position: $x_{n}=x_{n}+v_{n}$ 
Where $x_{n}$ and $v_{n}$ are the position and the speed of vehicle $n ; a$ is the acceleration.

\section{Simulation and Analysis of Model}

Simulation uses periodic boundary conditions, sets slow probability $P=0.2, P_{\text {change }}=0.7 ; a=1 ; v_{\text {max }}=3$, changes the traffic density and the probability of radical change to study traffic flow characteristics of electric bicycle. The simulation results are shown in Fig. 1-3.

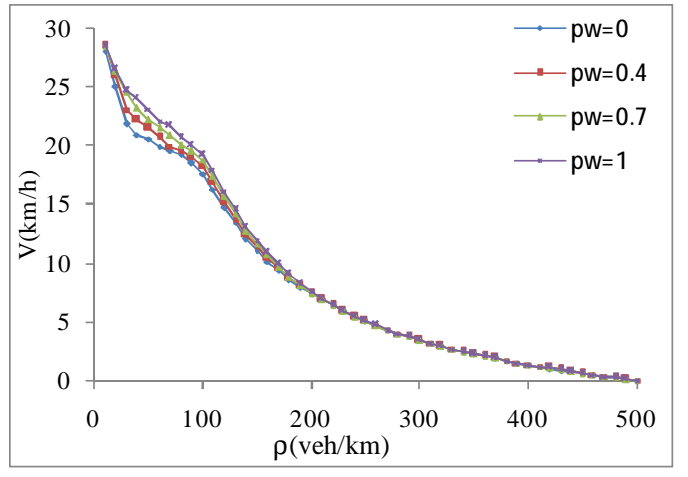

Figure 1. Curve of density-velocity

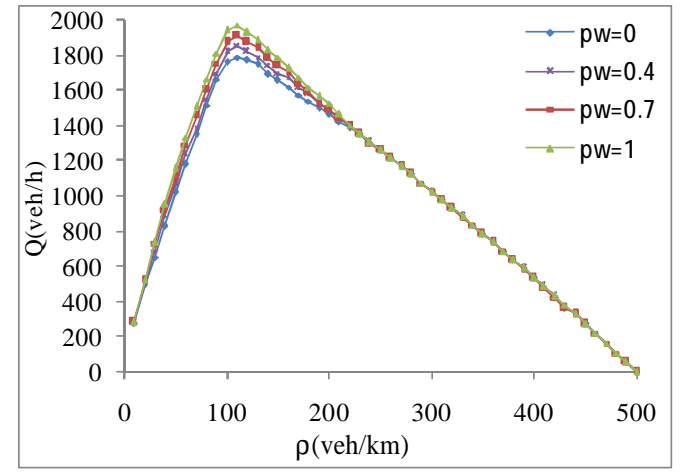

Figure 2. Curve of Density-flow

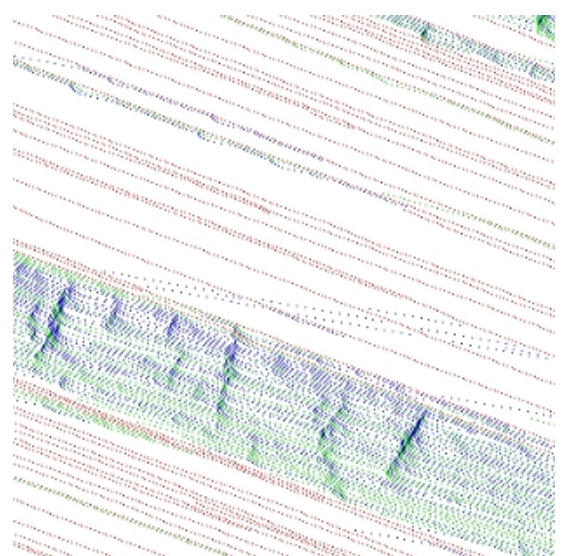

(b) Lane 2

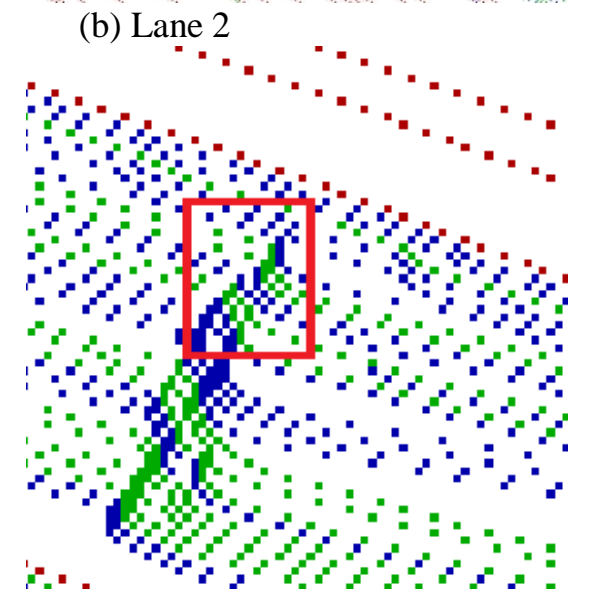

(d) Partial discharge of lane 3

with $p_{w}=0, p=0.2, p_{\text {change }}=0.7, \rho=30 \mathrm{veh} / \mathrm{km}$

(c) Lane 3

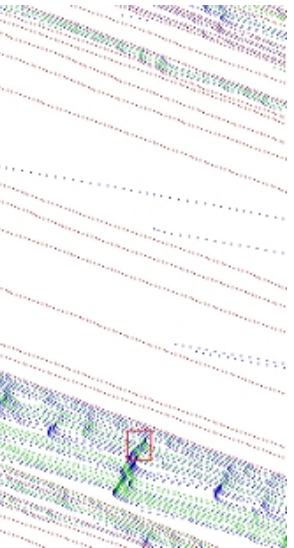

Fig. 1 and Fig. 2 shows respectively the density - speed curve and density - flow curve under different conditions of whistle probability and other simulation conditions are $P=0.2, P_{\text {change }}=P_{r}=0.7$. Figure 3-9 shows, in low density area, system average speed increases to a certain extent with the increase of whistle probability. When $\rho>200 \mathrm{veh} / \mathrm{km}$, the increase of whistle probability has no effect on 
the speed of the mixed traffic flow. As is shown in Fig. 2, in the range of $80 \mathrm{veh} / \mathrm{km}<\rho>220 \mathrm{veh} / \mathrm{km}$, with improvement of whistle probability, the mixed traffic flow has risen obviously, of which the maximum capacity is increased from $1791 \mathrm{veh} / \mathrm{h}$ when $p_{w}=0$ to $1980 \mathrm{veh} / \mathrm{h}$ when $p_{w}=1$. In other density range, the whistle probability has no effect on the mixed traffic flow.

Fig. 1 shows the temporal and spatial evolution figure of 3 lanes and 700-1000 cellulars under the condition of the probability $P_{w}=0$ whistle. Traffic flow drives from left to right, and each pixel in horizontal direction for each row represents a cellular, of which the white dots represent blank cellular, and the next line represents the locations of the vehicles at the next moment. When whistle probability $P_{w}=0.7,3$ lane space utilization rate is greatly reduced. As shown in Fig. 3(d) of partial discharge image of lane 3, as a result of low speed blocking in front, a large number of great speed vehicles have to slow down, resulting in a large number vehicles run at low speed.

\section{Conclusions}

According to the running characteristics of the electric bicycle and combining with the characteristics that different speed vehicles have different speed expectations, this paper divides electric bicycle flow change into general lane change, and whistle change, and establish the corresponding lane changing rules. Combined with the NS model, the electric bicycle traffic CA model is established and simulation analysis of the model in different whistle lane changing probability conditions has been carried out. The results show that: when the traffic density $\rho<220 \mathrm{veh} / \mathrm{km}$, whistling behavior has certain effect to improve road utilization rate and improve the road capacity. While in high density, whistle behavior can not improve the road capacity.

\section{Acknowledgments}

This work is partially supported by National Natural Science Foundation (51268017) and Research Project of Science and Technology of Education Department of Jiangxi ( GJJ13427).

\section{References}

[1] K. Nagel, M. Schreckenberg. A cellular automaton model for freeway traffic, Journal de physique I. 1992, 2(12): 2221-2229.

[2] J. Esser, M. Schreckenberg. Microscopic simulation of urban traffic based on cellular automata, International Journal of Modern Physics C. 1997, 8(05): 1025-1036.

[3] S. Maerivoet, B. De Moor. Cellular automata models of road traffic, Physics Reports. 2005, 419(1): $1-64$.

[4] Y. Taniguchi, H. Suzuki. A Traffic Cellular Automaton with Estimation of Time to Collision, J. Cellular Automata. 2013, 8(5-6): 407-416.

[5] M. Jing, W. Deng, Y.J. Ji, et al., Influences of time step and cell size on cellular automaton model, Journal of Jilin University(Engineering and Technology Edition). 2013, 43(2): 310-316.

[6] D. Zhao, W. Wang, C. Li, et al., Modeling of passing events in mixed bicycle traffic with cellular automata, Transportation Research Record: Journal of the Transportation Research Board. 2013 (2387): 26-34.

[7] J. Vasic, H.J. Ruskin, Agent-based space-time discrete simulation of urban traffic including bicycles, Procedia Computer Science. 2012, 10: 860-865.

[8] X. Chen, X. Fang, B. Lin, The Research on the Interaction between Mopeds and Bicycles Using Cellular Automata Method, Sensors \& Transducers. 2013, 161(12): 493. 